\title{
Cardio-Ankle Vascular Index (CAVI) and its Potential Clinical Implications for Cardiovascular Disease
}

\author{
Kazuhiko Kotani ${ }^{1}$ and Alan T Remaley ${ }^{2 *}$
}

${ }^{1}$ Department of Clinical Laboratory Medicine, Jichi Medical University, Shimotsuke-City, Tochigi 329-0498, Japan

${ }^{2}$ Cardiopulmonary Branch, National Heart Lung and Blood Institute, National Institutes of Health, Bethesda, MD 20892-1508, USA

\begin{abstract}
Arterial stiffness is a surrogate indicator for the development of cardiovascular disease. The cardio-ankle vascular index (CAVI) is a recently developed clinical metric for evaluating arterial stiffness. Two characteristics of $\mathrm{CAVI}$, which makes it amenable for widespread use, is its simplicity of measurement and the fact that it is relatively independent of blood pressure. Several recent clinical studies have shown the possible utility of CAVI for identifying patients at high risk for cardiovascular disease and for monitoring treatment effectiveness in lipid clinics.
\end{abstract}

Keywords: Atherosclerosis; Arterial stiffness; Cardiovascular disease; Dyslipidemia

\section{A History of Arterial Stiffness Evaluation}

Arterial stiffness is associated with atherosclerotic risk factors and is considered to be a surrogate marker for the development of cardiovascular disease [1-3]. Indeed, arterial stiffness occurs in patients with hypertension [4], diabetes mellitus [5], dyslipidemias [6], and obesity and metabolic syndrome [7-9], conditions or disease processes that are known to be pathophysiologically linked with cardiovascular disease. Based on its association with cardiovascular disease, it has been proposed that the evaluation and monitoring of arterial stiffness may be useful for determining cardiovascular disease risk and for monitoring therapy [10]. As will be described in this review, the cardio-ankle vascular index (CAVI) is a recently developed metric for evaluating arterial stiffness and for possibly predicting cardiovascular disease risk [11-13].

Several different methods have been developed for evaluating of arterial stiffness, such as carotid-femoral pulse wave velocity (cfPWV) [1-6,10], augmentation index (AI) [14], and stiffness parameter $\beta$ $[15,16]$. Of these, the most commonly used indicator is the cfPW. This measurement, however, is affected by blood pressure, an important confounding variable for cardiovascular disease [17]. In addition, cfPWV is often overestimated because of inaccuracies in the distance measurement between the carotid and the femoral to measure the pulse wave [18].

Heart-femoral PWV (hfPWV) is an alternative measure of arterial stiffness, which was developed to adjust for blood pressure $[19,20]$. This method is based on the correction of blood pressure, using a fixed diastolic pressure of $80 \mathrm{~mm} \mathrm{Hg}$ in order to reduce the influence of blood pressure $[19,20]$. The hfPWV is expressed by the following equation: hfPWV $=(\mathrm{D} \times 1.3) /(\mathrm{t}+\mathrm{tc})$, where ' $\mathrm{D}$ ' is the distance between the right edge of the sternum at the second intercostal space and the site of femoral pulsation, ' 1.3 ' is the anatomical correction value [17], ' $t$ ' is the time interval between the rise of the carotid pulse wave and the rise of femoral pulse wave, and ' $t c$ ' is the time interval between the second heart sound (S2) and the notch of the carotid pulse wave. The clinical significance of hfPWV has been confirmed $[21,22]$ but requires a high level of proficiency in order to obtain accurate results.

The stiffness parameter $\beta$, another measure of arterial stiffness, can be described by the following equation: stiffness parameter $\beta=\ln (\mathrm{Ps} /$
$\mathrm{Pd}) \times \mathrm{D} / \Delta \mathrm{D}$, where 'Ps' is the maximum (systolic) blood pressure, 'Pd' is the minimum (diastolic) blood pressure, 'D' is the diameter of the artery, and ' $\Delta \mathrm{D}$ ' is the change in arterial diameter caused by the difference in blood pressure [15]. Because this stiffness parameter $\beta$ represents the logarithmic change in blood pressure required to increase arterial diameter, it is relatively independent of blood pressure [15]. Yet, the use of the stiffness parameter $\beta$ remains problematic as it is obtained by assessing only a local segment of the artery, and it begins to lose its independence of blood pressure for individuals with moderate to severe hypertension or hypotension [15].

\section{Basis of CAVI}

To resolve the technical and practical issues of accurately measuring arterial stiffness, the cardio-ankle vascular index, CAVI, which incorporates the stiffness parameter $\beta[11,12,23]$, was developed. Devices, such as VaSera VS-1500AU, for measuring CAVI are very compact and portable, and their operation is relatively simple and not time-consuming unlike electrocardiography (ECG). Because the CAVI is dependent upon the stiffness parameter $\beta$, it is relatively unaffected by blood pressure $[11,12,23]$. Another unique feature of CAVI is the sites used for measuring CAVI. The pulse wave in the extremities is measured with changes in the air volume of blood pressure cuffs placed on the four extremities [11,12]. This obviates some of the problems related to the sites for measurement (i.e. femoral attachment in hfPWV and local evaluation of stiffness parameter $\beta$ ) and provides an overall arterial stiffness from the heart to the ankle. It also simultaneously measures the ankle-brachial index, another measure physiologic measure of vascular health.

The CAVI measurement requires the placement of ECG electrodes on both wrists, and a microphone for phonocardiography on the sternum in the second intercostal space, and 4 blood pressure cuffs

*Corresponding author: Alan T Remaley, Cardiopulmonary Branch, National Heart Lung and Blood Institute, National Institutes of Health, Bethesda, MD 20892 1508, USA, E-mail: aremaley1@cc.nih.gov

Received April 08, 2013; Accepted May 22, 2013; Published May 28, 2013

Citation: Kotani K, Remaley AT (2013) Cardio-Ankle Vascular Index (CAVI) and its Potential Clinical Implications for Cardiovascular Disease. Cardiol Pharmaco 2: 108. doi:10.4172/2329-6607.1000108

Copyright: @ 2013 Kotani K, et al. This is an open-access article distributed under the terms of the Creative Commons Attribution License, which permits unrestricted use, distribution, and reproduction in any medium, provided the original author and source are credited. 
wrapped around the four extremities. In this way, the upper arm and ankle pulse waves, as well as blood pressure, can all be measured using plethysmography. The coefficients of variation of CAVI are relatively small, typically $<4 \%$, and does not require significant training [11].

CAVI is calculated using PWV from the aortic valve origin to the ankle region and blood pressure measured at the upper arm (Figure 1). The formula for CAVI uses the Bramwell-Hill's equation, which represents the relationship between PWV and volume change [24] and is derived from the stiffness parameter $\beta$ first proposed by Hayashi et al. [15]. CAVI is calculated as follows: $\mathrm{CAVI}=\mathrm{a}\{(2 \rho / \Delta \mathrm{P}) \times \ln (\mathrm{Ps} / \mathrm{Pd}) \times$ $\left.\mathrm{PWV}^{2}\right\}+\mathrm{b}$ (Equation 1), where 'Ps' is the systolic blood pressure, 'Pd' is the diastolic blood pressure, 'PWV' is the pulse-wave velocity from the aortic origin to the ankle region via the femoral artery, ' $\Delta \mathrm{P}$ ' is Ps - Pd, ' $\rho$ ' is the blood viscosity, and 'a' and ' $b$ ' are constants for converting a CAVI value to a value obtained by Hasegawa's method $[19,20]$.

The equation of the stiffness parameter $\beta$ includes $\mathrm{D} / \Delta \mathrm{D}$, which can be obtained by modifying Bramwell-Hill's equation [24]: $\mathrm{PWV}^{2}$ $=\Delta \mathrm{P} / \rho \times \mathrm{V} / \Delta \mathrm{V}$ (Equation 2), where ' $\Delta \mathrm{P}$ ' is the pulse pressure, ' $\mathrm{V}$ ' is the blood vessel volume, ' $\Delta \mathrm{V}$ ' is the change in $\mathrm{V}$, and $\rho$ is the blood viscosity. ' $\mathrm{V} / \Delta \mathrm{V}$ ' can be expressed by ' $\mathrm{D}$ ' and ' $\Delta \mathrm{D}$ ', as shown below: $\mathrm{V} /$ $\Delta \mathrm{V}=\left(\pi \mathrm{L}(\mathrm{D} / 2)^{2}\right) /\left[\pi \mathrm{L}((\mathrm{D}+\Delta \mathrm{D}) / 2)^{2}-\pi \mathrm{L}(\mathrm{D} / 2)^{2}\right]=\mathrm{D}^{2} /\left[\mathrm{D}^{2}+2 \mathrm{D} \Delta \mathrm{D}+\right.$ $\left.\Delta \mathrm{D}^{2}-\mathrm{D}^{2}\right]=\mathrm{D}^{2} /\left(2 \mathrm{D} \Delta \mathrm{D}+\Delta \mathrm{D}^{2}\right)$. Because ' $\Delta \mathrm{D}^{2}$ ' is negligible compared with ' $2 \mathrm{D} \Delta \mathrm{D}$ ', it can be ignored. The equation thus becomes as follows: $\mathrm{V} / \Delta \mathrm{V}=\mathrm{D}^{2} / 2 \mathrm{D} \Delta \mathrm{D}=\mathrm{D} / 2 \Delta \mathrm{D}$ (Equation 3). Therefore, $\mathrm{V} / \Delta \mathrm{V}$ in Equation 2 can be replaced by $\mathrm{D} / 2 \Delta \mathrm{D}$. Equation 2 becomes as follows: $\mathrm{PWV}^{2}$ $=\Delta \mathrm{P} / \rho \times \mathrm{V} / \Delta \mathrm{V}=\Delta \mathrm{P} / \rho \times \mathrm{D} / 2 \Delta \mathrm{D}$, which can be rearranged as follows: $\mathrm{D} / \Delta \mathrm{D}=2 \rho / \Delta \mathrm{P} \times \mathrm{PWV}^{2}$ (Equation 4 ).

Thus, the new $\beta$ value can be obtained by substituting Equation 4 in the stiffness parameter $\beta$ equation by the following relationship: $\beta=$ $\ln (\mathrm{Ps} / \mathrm{Pd}) \times(\mathrm{D} / \Delta \mathrm{D})=\ln (\mathrm{Ps} / \mathrm{Pd}) \times 2 \rho / \Delta \mathrm{P} \times \mathrm{PWV}^{2}$ (Equation 1$)$. The new equation for the stiffness parameter $\beta$ obtained in this manner reflects the overall stiffness from the aorta, femoral artery, and tibial artery;

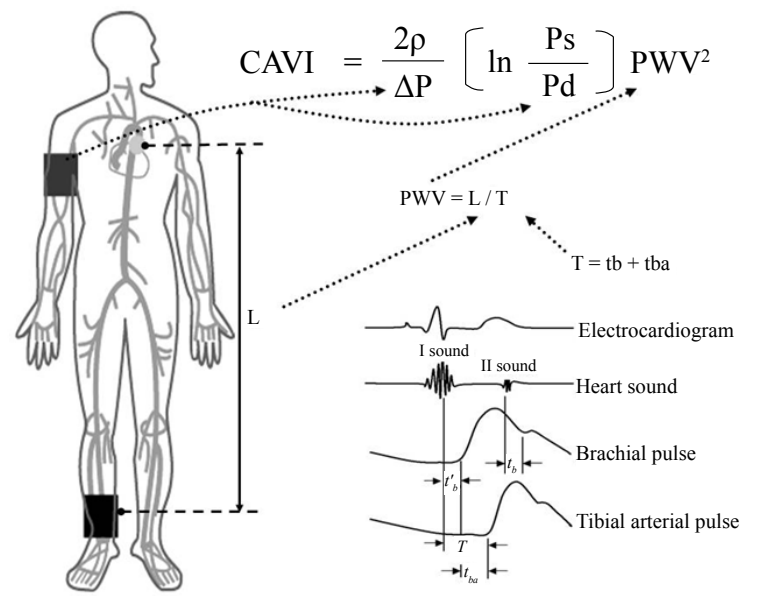

Figure 1: Principle of CAVI.

Pulse wave velocity (PWV) from the heart to the ankle is obtained by measuring the length from the origin of the aorta to the ankle, and by calculating $\mathrm{T}=\mathrm{tb}+\mathrm{tba}$ Blood pressure is measured at the brachial artery. Ps: systolic blood pressure, $\mathrm{Pd}$ : diastolic blood pressure, $\Delta \mathrm{P}$ : Ps-Pd, $\rho$ : blood density, $\triangle \mathrm{P}$ : pulse pressure, $\mathrm{L}$ : length from the origin of the aorta to the ankle, $\mathrm{T}$ : time taken for the pulse wave to propagate from the aortic valve to the ankle, tba: time between the rise of the brachial pulse wave and the rise of the ankle pulse wave, tb: time between aortic valve closing sound and the notch of brachial pulse wave, t'b: time between aortic valve opening sound and the rise of the brachial pulse wave. hence, the equation is named the "cardio-ankle vascular index (CAVI)." In theory, the CAVI has the characteristic of independence of blood pressure and is essentially intrinsic to the stiffness parameter $\beta$.

\section{Evidence of Independence of Blood Pressure}

Several clinical and experimental studies [11,12,25-28] have revealed that the CAVI is largely independent of blood pressure at the time of measurement, unlike PWV. For example, an experimental comparative study [28] was done by the studying the acute effect of several blood pressure modifying drugs, such as the $\beta_{1}$-adrenoceptor blocker metoprolol. Both CAVI and brachial-ankle PWV (baPWV) were measured every hour for 6 hours after administrating the drugs. In the metoprolol group, mean systolic blood pressure decreased from 131.4 to $118.3 \mathrm{mmHg}(P<.05)$ and mean diastolic blood pressure decreased from 85.3 to $75.3 \mathrm{mmHg}(P<.05)$. baPWV also decreased from 13.9 to $12.5 \mathrm{~m} / \mathrm{sec}(P<.05)$ after drug treatment, whereas CAVI did not change (8.2 to 8.2$)(P=.45)$. Similar results showing the independence of CAVI on blood pressure were also found after treatment with the $\alpha_{1}$ adrenoceptor blocker doxazosin (Figure 2) [28].

Statin treatment can also induce a mild blood pressure reduction [29] and this means that direct cholesterol-lowering effects of statins on arterial stiffness and vascular function may be unknown if the study groups have a significant blood pressure reduction. Therefore, CAVI is suitable in these cases.

\section{Reference Level of CAVI by Age and Gender}

In order to determine the reference values for CAVI, a large-scale study was conducted in Japan in healthy individuals free of cardiovascular disease [30]. In men, CAVI increased almost linearly with age, from 20 to 70 years. The CAVI level in men was higher than women for almost all age groups, but both men and women showed a similar rate of increase in CAVI of approximately 0.5 per year (Figure 3 ).
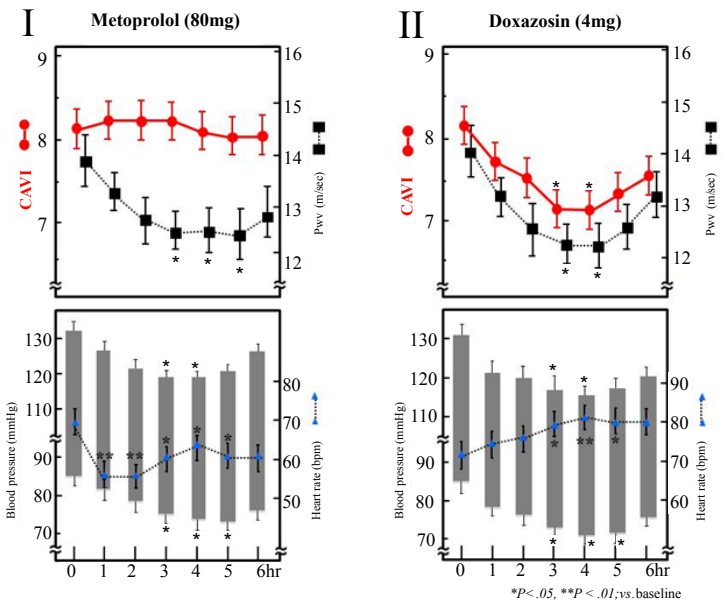

Figure 2: Effects of $\beta_{1}$ - and $\alpha_{1}$-blockers on blood pressure, PWV and CAVI. I: When a selective $\beta_{1}$-adrenergic blocker, metoprolol, was administered, both systolic and diastolic blood pressures decrease and PWV also decreases, but $\mathrm{CAVI}$ does not change.

II: When a selective $\alpha_{1}$-aderenergic receptor blocker, doxazosin, was administered, both systolic and diastolic blood pressures decreased. Both PWV and CAVI decrease. 
A potential cut-off level of CAVI for the diagnosis of coronary heart disease was also determined [31]. A cut-off level of CAVI of 8.67 yielded a sensitivity of $66.5 \%$ and a specificity of $65.8 \%$; thus a CAVI score of 9.0 is commonly used for the diagnosis of cardiovascular disease. It will be important, however, to establish the optimum cut-off in other populations and given the close relationship between CAVI and age (Figure 3), it may be best to use age-dependent values.

\section{Clinical Studies on Dyslipidemias using CAVI}

The association between atherosclerotic risk factors, including dyslipidemias, and CAVI has been investigated in several studies. For instance, the association between lipids and CAVI has been investigated in cross-sectional studies. One study found that the CAVI score significantly correlated with the LDL-cholesterol level $(r$ $=0.26, P<.05)$ in patients with angina [32]. The CAVI score was also found to be significantly increased with hypercholesterolemia and hypertriglyceridemia in men (of 30-69 years of age) and women (of 4075 years) in comparison to those without CVD risk factors [30].

Presently, there have been three intervention studies using CAVI for dyslipidemias. In one study, a highly purified eicosapentaenoic acid (EPA), an n-3 polyunsaturated fatty acid, was tested as an antiatherogenic treatment modality, along with CAVI [33]. In this randomized controlled intervention study, obese patients with metabolic syndrome $(\mathrm{n}=92 \quad[\mathrm{men} /$ women $=39 / 53]$, mean age $=51.7$ years, $\left.\mathrm{BMI}=30.0 \mathrm{~kg} / \mathrm{m}^{2}, \mathrm{HbA} 1 \mathrm{c}=6.3 \%\right)$ were treated with $\mathrm{EPA}(1.8 \mathrm{~g} /$ day) for 3 months [33]. When compared to the control group $(n=46$ [men/women $=19 / 27]$, mean age $=52.2$ years), the EPA-treated group $(n=46[\mathrm{men} /$ women $=20 / 26]$, mean age $=51.3$ years $)$ showed a significant reduction of mean levels of triglycerides (from 237 to $177 \mathrm{mg} / \mathrm{dL}$ versus from 227 to $198 \mathrm{mg} / \mathrm{dL}$ in the control, $P<.01$ ), C-reactive protein (from 1.57 to $1.16 \mu \mathrm{g} / \mathrm{mL}$ versus from 1.46 to $1.63 \mathrm{mg} / \mathrm{dL}$ in the control, $P<.01)$ and serum amyloid A-LDL complex, a marker of inflammation and oxidative stress (from 48.6 to $40.4 \mu \mathrm{g} / \mathrm{mL}$ versus from 47.5 to 51.2 $\mathrm{mg} / \mathrm{dL}$ in the control, $P<.01)$. In this study, the CAVI score also showed a modest decrease (from 7.87 to 7.59 versus from 7.76 to 7.80 in the control, $P<.01)$. A multivariate linear regression analysis revealed that the only significant determinant for a reduction in CAVI by EPA was a reduction in serum amyloid A-LDL complex $(\beta=0.38, P<.05)$.

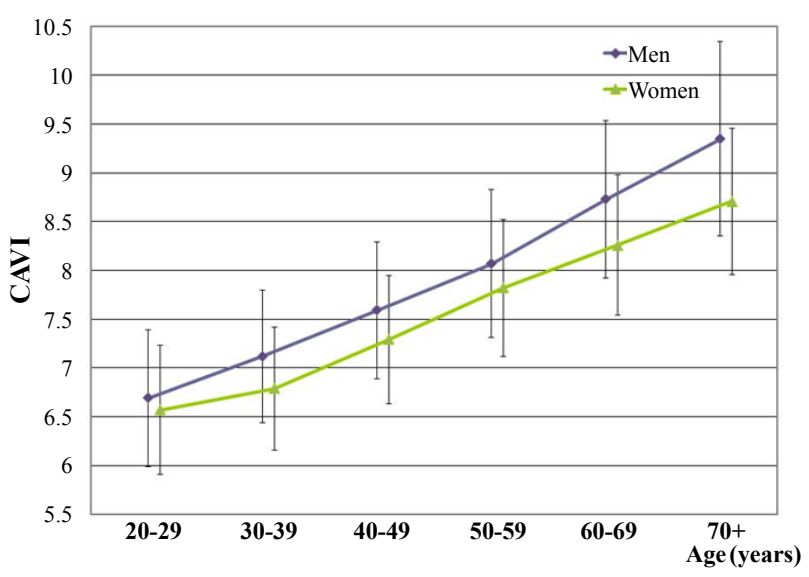

Figure 3: CAVI by age and gender.

The CAVI level increases with age almost linearly from 20 to 70 years in males and females by almost 0.5 over 10 years. The CAVI level of males is higher than that of females in all age groups.

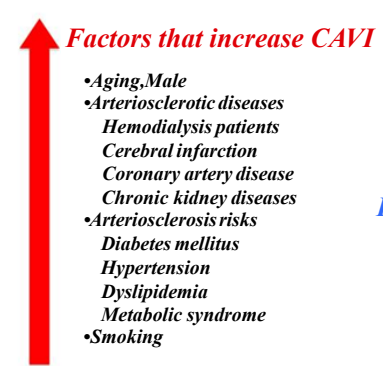

Factors that decrease CAVI

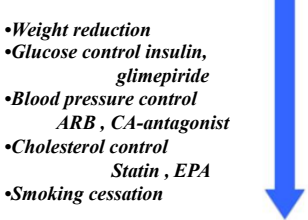

Figure 4: Factors associated with CAVI.

Excess cholesterol absorption in the small intestine has also been suggested to be a risk factor of atherosclerosis. A single-arm intervention study investigated the effect of monotherapy of ezetimibe $(10 \mathrm{mg} /$ day) for 6 months on CAVI in patients with type 2 diabetes mellitus $(\mathrm{n}=45$ [men/women=23/17], mean age $=64$ years, $\mathrm{HbAlc}=6.5 \%)$ [34]. After ezetimibe treatment, significant decreases in mean levels of serum LDL-C (from 160 to $123 \mathrm{mg} / \mathrm{dL}, P<.01$ ) and CAVI (from 9.17 to $9.00, P<.05)$ were observed. Further sub analysis divided patients into the two groups that achieved the LDL-C level of $<120 \mathrm{mg} / \mathrm{dL}$ as a treatment goal by ezetimibe $(n=15[\mathrm{men} /$ women $=10 / 5]$, mean age $=65$ years, $\mathrm{HbA} 1 \mathrm{c}=6.7 \%$, at-baseline LDL-C $=157 \mathrm{mg} / \mathrm{dL}$ ) and that did not achieve the LDL-C level of $<120 \mathrm{mg} / \mathrm{dL}$ ( $\mathrm{n}=25$ [men/women=13/12], mean age $=63$ years, $\mathrm{HbA} 1 \mathrm{c}=6.3 \%$, at-baseline $\mathrm{LDL}-\mathrm{C}=163 \mathrm{mg} / \mathrm{dL})$. A significant reduction of mean CAVI levels (from 9.52 to $9.20, P<.05$ ) was observed in the group that achieved the LDL-C level of $<120 \mathrm{mg} /$ $\mathrm{dL}$, but not the group that did not achieve the LDL-C level of $<120 \mathrm{mg} /$ $\mathrm{dL}$ (from 8.95 to 8.86 ). This result suggests that the group that achieved a greater LDL-C reduction might have excess cholesterol absorption, possibly leading to a high CAVI level.

In addition to their cholesterol-lowering effects, statins can have effects in reducing oxidative stress, which has also been examined by CAVI [35]. A single-arm intervention study investigated the effect of pitavastatin ( $2 \mathrm{mg} /$ day) for 12 months on CAVI in patients with type 2 diabetes mellitus $(n=45$ [men/women $=19 / 26]$, mean age $=66$ years, $\mathrm{HbAlc}=6.9 \%$ ) [35]. After pitavastatin treatment, significant decreases in mean levels of serum LDL-C (from 166 to $127 \mathrm{mg} / \mathrm{dL}, P<.01$ ), MDALDL, a marker of oxidatively modified LDL (from 170 to $114 \mathrm{U} / \mathrm{L}$, $P<.01$ ), and CAVI (from 9.54 to $8.91, P<.05$ ) were observed. Further correlation analysis revealed that the change of CAVI was significantly and positively correlated with that of MDA-LDL $(r=0.55, P<.05)$, but not that of LDL-C, during a pre- and post-treatment period.

\section{Perspectives on CAVI}

In summary, CAVI is a new non-invasive indicator of arterial stiffness. Its main advantages over other procedures for measuring arterial stiffness are its independence on blood pressure and its relative simplicity and low cost. As summarized in Figure 4, CAVI has been shown to correlate with a wide variety of factors and disease conditions that are known to be associated with cardiovascular disease [36]. Given the fact that the subjects that develop myocardial infarction do not always appear to be risk based on conventional markers [37], such as lipid and lipoprotein tests, it makes CAVI a potentially useful way for identifying patients at risk for cardiovascular disease and for possibly monitoring therapy that otherwise may be missed by current 
Citation: Kotani K, Remaley AT (2013) Cardio-Ankle Vascular Index (CAVI) and its Potential Clinical Implications for Cardiovascular Disease. Cardiol Pharmacol 2: 108. doi:10.4172/2329-6607.1000108

approaches. The clinical utility, however, of measuring CAVI as a cardiovascular risk marker has yet to be definitively established in large prospective clinical trials.

\section{Acknowledgement}

This work was supported by intramural NIH funds from NHLBI.

\section{References}

1. Asmar R (1999) Pulse wave velocity principles and measurement. In: Arterial stiffness and pulse wave velocity. Elsevier, Amsterdam 25-55.

2. Oliver JJ, Webb DJ (2003) Noninvasive assessment of arterial stiffness and risk of atherosclerotic events. Arterioscler Thromb Vasc Biol 23: 554-566.

3. Mattace-Raso FU, van der Cammen TJ, Hofman A, van Popele NM, Bos ML, et al. (2006) Arterial stiffness and risk of coronary heart disease and stroke: the Rotterdam Study. Circulation 113: 657-663.

4. O'Rourke MF, Staessen JA, Vlachopoulos C, Duprez D, Plante GE (2002) Clinical applications of arterial stiffness; definitions and reference values. Am J Hypertens 15: 426-444.

5. Koizumi M, Shimizu H, Shimomura K, Oh-I S, Tomita Y, et al. (2003) Relationship between hyperinsulinemia and pulse wave velocity in moderately hyperglycemic patients. Diabetes Res Clin Pract 62: 17-21.

6. Avest E, Holewijn S, Bredie SJ, van Tits LJ, Stalenhoef AF, et al. (2007) Pulse wave velocity in familial combined hyperlipidemia. Am J Hypertens 20: 263269.

7. Satoh N, Shimatsu A, Kato Y, Araki R, Koyama K, et al. (2008) Evaluation of the cardio-ankle vascular index, a new indicator of arterial stiffness independent of blood pressure, in obesity and metabolic syndrome. Hypertens Res 31: 19211930.

8. Liu H, Zhang X, Feng X, Li J, Hu M, et al. (2011) Effects of metabolic syndrome on cardio-ankle vascular index in middle-aged and elderly Chinese. Metab Syndr Relat Disord 9: 105-110.

9. Satoh-Asahara N, Suganami T, Majima T, Kotani K, Kato Y, et al. (2011) Urinary Cystatin $\mathrm{C}$ as a potential risk marker for cardiovascular disease and chronic kidney disease in patients with obesity and metabolic syndrome. Clin J Am Soc Nephrol 6: 265-273.

10. Tanaka H, Munakata M, Kawano Y, Ohishi M, Shoji T, et al. (2009) Comparison between carotid-femoral and brachial-ankle pulse wave velocity as measures of arterial stiffness. J Hypertens 27: 2022-2027.

11. Shirai K, Utino J, Otsuka K, Takata M (2006) A novel blood pressureindependent arterial wall stiffness parameter; cardio-ankle vascular index (CAVI). J Atheroscler Thromb 13: 101-107.

12. Shirai K, Hiruta N, Song M, Kurosu T, Suzuki J, et al. (2011) Cardio-ankle vascular index (CAVI) as a novel indicator of arterial stiffness: theory, evidence and perspectives. J Atheroscler Thromb 18: 924-938.

13. Kubota Y, Maebuchi D, Takei M, Inui Y, Sudo Y, et al. (2011) Cardio-Ankle Vascular Index is a predictor of cardiovascular events. Artery Research 5: 9196.

14. Lemogoum D, Flores G, Van den Abeele W, Ciarka A, Leeman M, et al. (2004) Validity of pulse pressure and augmentation index as surrogate measures of arterial stiffness during beta-adrenergic stimulation. J Hypertens 2: 511-517.

15. Hayashi K, Handa H, Nagasawa S, Okumura A, Moritake K (1980) Stiffness and elastic behavior of human intracranial and extracranial arteries. J Biomech 13: $175-184$

16. Kawasaki T, Sasayama S, Yagi S, et al. (1987) Noninvasive assessment of the age related changes in stiffness of major branches of the human arteries. Cardiovascular Res 21: 678-687.

17. Nye ER (1964) The effect of blood pressure alteration on the pulse wave velocity. Br Heart J 266: 261-265.

18. Van Bortel LM, Laurent S, Boutouyrie P, Chowienczyk P, Cruickshank JK, et al.
(2012) Expert consensus document on the measurement of aortic stiffness in daily practice using carotid-femoral pulse wave velocity. J Hypertens 30: 445-448.

19. Hasegawa M (1970) Fundamental research on human aortic pulse wave velocity. Jikei Medical Journal 85: 742-760.

20. Hasegawa M, Arai C (1995) Clinical Estimation of vascular elastic function and practical application. Connective Tissue 27: 149-157.

21. Hamazaki T, Urakaze M, Sawazaki S, Yamazaki K, Taki H, et al. (1988) Comparison of pulse wave velocity of the aorta between inhabitants of fishing and farming villages in Japan. Atherosclerosis 73: 157-160.

22. Saito Y, Shirai K, Utino J, Okazaki M, Hattori Y, et al. (1990) Effect of nefedipine administration on pulse wave velocity of chronic hemodialysis patients -2 years trial. Cardiovas Drugs Ther 4: 987-990.

23. Kotani K, Miyamoto M, Taniguchi N (2010) Clinical significance of the cardioankle vascular index (CAVI) in hypertension. Curr Hypertens Rev 6: 251-253.

24. Bramwell JC, Hill AV (1922) Velocity of the pulse wave in man. Proc Roy Soc B 93: 298-306.

25. Takaki A, Ogawa H, Wakeyama T, Iwami T, Kimura M, et al. (2007) Cardioankle vascular index is a new noninvasive parameter of arterial stiffness. Circ J 71: 1710-1714

26. Kubozono T, Miyata M, Ueyama K, Nagaki A, Otsuji Y, et al. (2007) Clinica significance and reproducibility of new artrial dstensibility index. Circ J 71: 8994.

27. Ibata J, Sasaki H, Kakimoto T, Nagaki A, Otsuji Y, et al. (2008) Cardio-ankle vascular index measures arterial wall stiffness independent of blood pressure. Diabetes Res Clin Pract 80: 265-270.

28. Shirai K, Song M, Suzuki J, Kurosu T, Oyama T, et al. (2011) Contradictory effects of $\beta_{1}$ - and $\alpha_{1}$-aderenergic receptor blockers on cardio-ankle vascular stiffness index (CAVI) - the independency of CAVI from blood pressure. $J$ Atheroscler Thromb 18: 49-55.

29. Kanaki Al, Sarafidis PA, Georgianos PI, Stafylas PC, Kanavos K, et al. (2012) Low-dose atorvastatin reduces ambulatory blood pressure in patients with mild hypertension and hypercholesterolaemia: a double-blind, randomized placebo-controlled study. J Hum Hypertens 26: 577-584.

30. Namekata T, Suzuki K, Ishizuka N, Shirai K (2011) Establishing baseline criteria of cardio-ankle vascular index as a new indicator of arteriosclerosis: a crosssectional study. BMC Cardiovasc Disord 11: 51.

31. Horinaka S, Yabe A,Yagi H, Ishimura K, Hara H, et al. (2009) Comparison of Atherosclerotic indicators between cardio ankle vascular index and brachia ankle pulse wave velocity. Angiology 60: 468-476.

32. Takaki A, Ogawa H, Wakeyama T, Iwami T, Kimura M, et al. (2008) Cardioankle vascular index is superior to brachial-ankle pulse wave velocity as an index of arterial stiffness. Hypertens Res 31: 1347-1355.

33. Satoh N, Shimatsu A, Kotani K, Himeno A, Majima T, et al. (2009) Highly purified eicosapentaenoic acid reduces cardio-ankle vascular index in association with decreased serum amyloid A-LDL in metabolic syndrome. Hypertens Res 32: 1004-1008.

34. Miyashita Y, Endo K, Saiki A, Ban N, Nagumo A, et al. (2010) Effect of ezetimibe monotherapy on lipid metabolism and arterial stiffness assessed by cardioankle vascular index in type 2 diabetic patients. J Atheroscler Thromb 17 1070-1076.

35. Miyashita Y, Endo K, Saiki A, Ban N, Yamaguchi T, et al. (2009) Effects of pitavastatin, a 3-hydroxy-3-methylglutaryl coenzyme a reductase inhibitor, on cardio-ankle vascular index in type 2 diabetic patients. J Atheroscler Thromb 16: $539-545$.

36. Shirai K, Utino J, Saiki A, Tatsuno I, Shimizu K, et al. (2012) Evaluation of arteriosclerotic vascular disease with a new noble stiffness indicator, cardioankle vascular index (CAVI). J Clin Exp Cardiolog S1-004.

37. Dominiczak MH (2001) Risk factors for coronary disease: the time for a paradigm shift? Clin Chem Lab Med 39: 907-919. 